\title{
PRAXIS OF CLIMATE CHANGE ADAPTATION IN DISASTER RISK REDUCTION: A SOUTH AFRICAN PERSPECTIVE
}

\author{
LOGANATHAN MUNSAMY \\ Department Government Management, Central University of Technology, South Africa
}

\begin{abstract}
Global warming is the rising of the earth's temperature due to increased anthropogenic greenhouse gas (GHG) emissions resulting in climate change and causing extreme erratic weather. Furthermore, the adverse effects of climate change are an increase in poverty levels causing large scale death and destruction. Global climate change must be adequately addressed in accordance with international protocols if developmental goals are to be achieved. In addition to a literature review, the empirical study used a mixed approach with a pragmatic paradigm. Data sets were processed using the Statistical Package for Social Sciences (SPSS 24), achieving a coefficient correlation of 0.74 using the Cronbach's alpha as a test measure. This study found that the existing disaster risk reduction (DRR) policies must be revised and amalgamated to ensure that development becomes sustainable; secondly, climate change adaptation (CCA) programs must be located within the appropriate government structures; thirdly, an effective climate change forum must be established; and finally, community structures must be strengthened to provide effective support for climate change policy development. South Africa is a fossil-fuelled energy-intensive, and relative to its economy, a major contributor to GHG emissions in Africa and its CCA policies are still in its infancy stages. For this reason, it needs a common national data collection instrument to measure carbon emissions and the impact thereof. Furthermore, there must be adequate political support for an effective monitoring and evaluation instrument to collect and analyse data to generate new knowledge regarding climate change and DRR as a single entity.
\end{abstract}

Keywords: climate change adaptation, disaster risk management, disaster risk reduction, global warming.

\section{INTRODUCTION}

Global warming is one of the main causes of climate change throughout the world causing extreme erratic weather patterns, resulting in hydrometeorological hazards. These hydrometeorological hazards, namely floods, fires, droughts and severe storms are the main cause of much death and destruction throughout the world, affecting mainly poor communities who are the most vulnerable. For this reason, extensive climate change adaptation (CCA) research has been carried out with many susceptible communities in countries such as Bangladesh, Pacific Island of Kiribati, Gambia in North Africa, and Southern African Development Community. In fact, the United Kingdom is leading in climate change research which includes interviews with more than 252 experts and scientists on global warming and consequently climate change which has a negative influence on lives and infrastructure. Since, global warming is exacerbated by human behaviour, it is anthropogenic and requires a concerted effort by all governments to implement effective behaviour modification programmes to limit the impact of climate change and consequently global warming. If the negative impact of extreme weather which is caused by climate change is minimised, then behaviour change of communities must be considered a priority by all governments.

Thus, this paper is prepared into six sections. The first section discusses the introduction and provides for the background to the problem. The second section presents an overview of the conceptualisation of disaster risk management (DRM) and climate change. In the third 
section a discourse on the methodology for this research paper is presented. The fourth section presents an analysis and discussion of the results and the arguments for the praxis of climate change in the context of DRM. This is followed by the conclusion undertaken for this research which advocates for the deliberate implementation of behaviour modification programmes to promote CCA as an important climate change response strategy for South Africa. In addition, the effectiveness of this programme will be enhanced if it is supported by an effective monitoring, evaluation and reporting mechanism.

\section{BACKGROUND (DISCUSSING THE PROGRESS IN THE LEGISLATION)}

Since the advent of democracy in South Africa (SA), significant progress has been made in reforming the DRM legislation such as the Disaster Management Act (DMA) 57 of 2002, the National Disaster Management Framework (NDMF) of 2005, and the Disaster Management Amendment Act 16 of 2015. The previously fragmented, post-disaster, search, rescue and recovery approach to DRM has progressed into a formidable, proactive, pre-disaster planning system. Whereas the South African DMA (57 of 2002) refers to the internationally accepted DRM as a concept, countries leading in DRM still refer to the outdated concept, Disaster Management (DM). Van der Waldt et al. [1] affirm that the SA definition as cited in the DMA, (57 of 2002), focuses on a multisectoral and multidisciplinary approach which describes DRM as a continuous, integrated process of planning and implementing measures to prevent or reduce disasters risks; mitigate the severity or impact of disasters; plan for emergency preparedness; rapidly and effectively respond to disasters; and to make provision for post-disaster recovery and rehabilitation measures. Furthermore, Vermaak and Van Niekerk [2] emphasise that the increasing number of disasters necessitates an approach from beyond "disaster management" to DRM.

This approach recommends the development of mitigation and prevention strategies after conducting a risk assessment. Thus, Coburn and Promonis [3], argue that DRM refers to all aspects of planning and responding to disasters before, during and after the disaster. Although the SA DRM legislative framework is highly rated in the international DRM arena because of its human rights approach, the implementation thereof needs to improve [1], [4].

The South African Disaster Management Amendment Act (2015) defines climate change as the state of the climate that is identified by changes in the variability of its properties that continue for a few decades or even longer, and which is caused by natural and human activity. Furthermore, Wisner et al. [5] argue that climate change encompasses global environmental changes as a result of greenhouse gases (GHG) build-up into the atmosphere, leading to an increase in the mean atmospheric temperature and which contributes to the fluctuations in rainfall patterns in prevailing climates around the world. Global environmental change also refers to the impact of human activities which causes changes in the biosphere, such as sea level rise, inappropriate land-use and land-cover [5].

Although there are vast differences between CCA and DRR, communities have been adapting to climate change for centuries possessing a wealth of knowledge between "adapting to change" and "dealing with disaster" [6]. In addition, the United Nations Development Programme (UNDP) [7], Adaptation Policy for Climate Change: Developing Strategies, Policies and Measures, stipulate four main methods for analysing climate change-related challenges which are firmly embedded in the DRM milieu. Firstly, it is a hazard-based approach; secondly, a vulnerability-based approach; thirdly, an adaptive-capacity-based approach; and fourthly, a policy-based approach. Since all four approaches for analysing climate change-related challenges are rooted deeply in a DRM context, it may be argued that CCA and DRR strategies are inextricably linked [7]. 


\section{METHODS/DATA SOURCES}

An intensive literature review included national and international legislative frameworks and protocols (discussed elsewhere in this paper) was carried out to understand what previous researchers found regarding climate change and disaster risk reduction (DRR). Thus, the content from the literature review formed the basis for developing the questions for the empirical studies. Twenty-five semi-structured interviews were conducted with DRM and CCA practitioners representing seven entities; and 125 responses were received from the 171 officials employed in the same seven entities involving the quantitative approach. This postpositivistic paradigm validated the gaps generated by the positivistic analysis in order to verify the depth of the identified factors findings [8]. Thereafter, the various discourses were described and discussed; and the findings of the research was also analysed, interpreted, described and discussed. Finally, there was analytical reading, the synthesising of ideas and reworking of theories [9].

According to Gliem and Gliem [10], the Cronbach's alpha is a statistical test that splits the questions in different ways and calculates values for each of them. This value is called the coefficient correlation or the reliability coefficient, which normally ranges between 0 and 1.0. There is no lower limit to the coefficient and the closer it is to 1.0, the greater the reliability.

For this study, four items in the questionnaire were used to achieve internal reliability and consistency. The reliability coefficient for this study ranges from 0.63 to 0.86 as indicated in Table 2.

Table 2 indicates the items tested for internal consistency and the respondent's coefficient achieved. Thus, the Cronbach's alpha for this study is 0.74 [10].

Table 1: Interpreting internal consistency using Cronbach's alpha [10].

\begin{tabular}{lc}
\hline Internal consistency & Cronbach's alpha \\
\hline Excellent: & $<0.9$ \\
Good: & $<0.8$ \\
Acceptable: & $<0.7$ \\
Questionable: & $<0.7$ \\
Poor: & $<0.5$ \\
Unacceptable: & $<0.4$ \\
\hline
\end{tabular}

Table 2: Cronbach's (alpha) coefficient: DRM questionnaire. (Source: Researcher's own interpretation.)

Questionnaire section

Rate the functioning of DRM in your municipality

Rate the level of compliance of your institution with the Disaster Management Act of 2002

Rate the DRM services provided by the centre with which you are mostly involved

Rate the functioning of the DRM assessment forum with which you are involved
Respondents (alpha) coefficient

0.86

0.82

0.67

0.63 


\section{DISCUSSION}

\subsection{South African DRM}

Floods are the main type of disasters experienced by South Africans, with 25 major floods occurring since 1980. Moreover, between 2009 and 2010, all nine provinces and 28 municipalities were declared disaster areas as a result of flooding, 40 lives lost, and more than 20,000 people displaced. According to the South African Local Government Association (SALGA), which is the official representative of the municipalities, 92 of the 278 municipalities (33\%) did not budget for DRM services in 2011 which is a clear indication of the lack of capacity to render effective DRM services in SA [11].

\subsection{DRM and CCA are inextricably linked}

Whilst, DRM may be considered a continuous problem, CCA is an emerging challenge for countries around the world [5]. Furthermore, climate change poses a two-fold threat to DRM, firstly, it increases the intensity and frequency of weather-related hazards, and secondly, it decreases the coping capacity of communities because developing countries will have to contend with reduced food and water supplies, and medical facilities [12], [13]. Therefore, it would be beneficial if there is convergence between both fields of study, especially in developing countries where they are inextricably linked at grassroots level [6].

\subsection{Climate change response strategy}

The World Resource Institute [14] defines CCA as the progression of changing behaviour from actual or predicted climatic changes in the community and the environment.

The National Climate Change Response Strategy (NCCR) [15] refers to CCA as a change in the behaviour of vulnerable communities to minimise the impact of weather-related disasters [14], [15]. When exposed to harsh climatic conditions, vulnerable communities may seek refuge in adaptive behaviours such as crop diversification, indigenous famine warning system and increasing water storage capacity [14], [16].

Thus, following a severe drought (2003-2005), a small group of farmers in the Northern Cape Province initiated the Highveld co-operative to improve their livelihood from frequent, severe droughts and extreme weather conditions [17].

The two main objectives of South Africa's NCCR initiative (2011) to climate change are, firstly, to effectively manage climate change hazards; and secondly, to contribute towards stabilising GHG emissions. Since, the net result of implementing sustainable CCA strategies are the reduction of disaster risks [15], the NCCR White Paper (2011) recommends five collaborative adaptation strategies; firstly, to use a national GHG emissions monitoring and evaluation (M\&E) system; secondly, to define desired emission reduction outcomes for each sector; thirdly, to adopt a carbon budget approach and submitting carbon-emissions reduction plans by sectors; fourthly, to developing a national GHG emission data inventory, and lastly, to develop an effective M\&E system [15].

\subsection{Monitoring and evaluation in DRR}

\subsubsection{Generic M\&E instruments}

Climate change and weather-related disasters affect different communities differently [11] and therefore, the use of generic M\&E instruments may not be effective in every situation. For instance, Namibia, Zimbabwe, Botswana, Tanzania and Zambia, belong to the Southern 
African Development Community but are all affected differently by weather patterns. Thus, Namibia, Zimbabwe and Botswana are arid and more affected by drought than Tanzania and Zambia which are more humid. Although all these countries are vulnerable to climate change and weather-related disasters, some are more affected by drought whilst others by floods.

Therefore, using generic M\&E instruments to measure the impact of climate change for all Southern African Development countries would not be appropriate [18]. For the M\&E instrument to serve its purpose adequately [18] explains that the instrument must contain indicators that best suit local climatic conditions. What works in one community may not work as well in another due to variations in local weather conditions [18].

\subsubsection{M\&E instruments}

The World Resources Institute [19] argues that M\&E in CCA serves two important purposes, firstly, $M \& E$ instruments may be used to understand climate change better; and secondly, as a data and reporting instrument to ensure effectiveness of climate change programmes. These two purposes are outlined below.

M\&E as a learning instrument should be used as the first step to understand CCA better because practitioners may use it as an instrument to collect, analyse, evaluate and report their findings. For instance, the United Nations Framework Convention on Climate Change (UNFCCC) [20] developed a five-year Nairobi Work Programme on impacts, vulnerability and adaptation which aims to assist least developed countries and small island developing states to improve their understanding of CCA strategies. It is used to distribute the latest scientific and technical CCA research information. By using this five-year Nairobi Work Programme as a platform, countries will benefit from the valuable information it provides to make informed decisions to influence climate change policies [14], [20].

The M\&E instrument may also be used to collect data for purposes of evaluating CCA programmes since research related to global warming and CCA is on the increase [19]. Furthermore, [12] asserts that the increase in the global warming is constantly bringing about a new understanding of what constitutes climate change. For this reason, some indicators in the M\&E instrument may become redundant and obsolete as new indicators may have to be included [12], [19].

As an illustration, the water flow of the Nile River is estimated to increase by $30 \%$ in wetter conditions, and to reduce to half in drier conditions. This is due to the anticipated and future predictions on climate change [19]. Thus, the construction of dams and reservoirs based on the current understanding of weather patterns may not apply in the long term as indicated by the impact of the weather on the Nile River [12], [19].

Although M\&E instruments are used to collect data to mitigate against severe weather patterns the envisaged increase or decrease in the flow of the Nile River will allow communities to adapt to these changes. Therefore, communities reliant on the Nile's reduced flow will become more resilient and their coping mechanism will increase to adapt and/or compete for scarce water resources [19]. This situation allows for a better understanding of CCA which will assist in developing effective strategies and programmes to mitigate against climate change.

The lesson for SA is to develop adaptation programmes such as increasing the number and size of water storage reserves and introducing crop varieties that can withstand extreme weather conditions. In addition, disaster prevention measures (CCA) may be integrated into regional planning, especially for critical infrastructure that relies heavily on water.

However, the uncertainty of weather patterns has brought about greater interest in the M\&E of CCA programme as indicated in the Fourth Assessment Report of the Intergovernmental Panel on Climate Change [21] which created a worldwide surge of 
interest in the implementation of CCA programmes. For purposes of sustainability the effectiveness of monitoring and evaluating CCA programmes must be community based (idiographic) as opposed to, government-centred (nomothetic) because the worst affected and most vulnerable communities, who are poor and lack political access, have little or no inputs in development that affects them directly. This untenable situation of lack of resources and access to support allows the poor to develop their own strategies for survival. For this reason, community-based initiatives must be nurtured and incorporated into government programmes that is developed by experts and scientists [21].

The next section will provide recommendations on how challenges related to CCA practices in South African municipalities can be overcome.

\subsubsection{Political commitment}

Political support represents a long-term investment on human and financial resources to plan prepare and limit the impact of climate change on communities and infrastructure [22]. As an example, Mozambique experienced severe flooding in 2000 losing 600 lives and causing US\$450 million of infrastructure damage. A flood of similar magnitude was experienced in 2007 where there was a significant reduction in the loss of life and property was due to the strong political will and commitment exercised by the presidency of Mozambique to ensure that the DRM functions are managed effectively [23].

International legislation such as the Hyogo Framework for Action (HFA) [24]; African Regional Strategy for DRR and its Plan of Action [25]; the Draft South African Development Community (SADC) Regional DRR Strategy [26]; and the SA NDMF [27] strongly stress the importance of political will in reducing disaster risks and climate change. One of the many ways in which politicians may intervene to assist in the reduction of disaster losses is by accelerating critical decision making [26]. In this regard, the example set by the President of the Maldives who led a team of experts, practitioners, policy-makers and political heavy weights to host a Leader's Forum to secure CCA and DRR firmly on the global agenda [12]. The President of the Philippines who intervened by heading the Climate Change Commission, which is the only policy-making body in his country linking every sector and department in the Philippines to ensure that disaster risks are reduced, critical infrastructure are protected, and development becomes sustainable.

These unprecedented examples of political will and commitment should be followed by other politicians to ensure that CCA and DRR strategies are in place to reduce disaster risks globally [12]. Although significant policy advances have been made in the field of DRR and climate change in SA, it is still plagued by a lack of finances and administrative capacity [27], [28]. A high-level political intervention will facilitate intergovernmental relations and cross-sectoral collaboration by assisting to overcome institutional barriers. Therefore, strong political will and commitment are significant factors in the successful implementation of climate change responses and DRR [12].

\section{RECOMMENDATIONS FOR EFFECTIVE CLIMATE CHANGE}

\subsection{Recommendation 1: Revise existing policies}

It is recommended that some existing climate change policies such as the Hazardous Substance Amendment Act (Act 53 of 1992) which causes ill health and/or deaths due to the corrosive, flammable or toxic nature of chemical substances that affects human beings and the environment. Since SA is bound by international agreements such as the provisions of Articles 4, 5, 6 and 12 of the UNFCCC; as well as Article 10 of the Kyoto Protocol which 
requires SA to develop, publish and regularly update policies to mitigate against climate change and disaster risks to avoid a negative impact on communities and critical infrastructure [29].

Although existing international protocols and agreements are an attempt to contribute to a globally sustainable world, the initiative to reduce global warming and disaster risks is the responsibility of all countries [12], [22]. However, one of the most important causes of pastoral mobility is climate change which influences the migratory patterns causing major conflicts such as in East Africa where herding is the main source of income, regular migration takes place. However, The International Federation of Red Cross and Red Crescent asserts that it is the responsibility of national governments to establish and maintain conducive regulatory policy environment for seamless implementation of climate risk assessment policies that are multi-sectoral and multi-dimensional in nature [18], [29], [31].

\subsection{Recommendation 2: Centralisation of monitoring and evaluation function of climate change programmes}

It is also recommended that the M\&E function of CCA as a risk reduction strategy be centralised. Since interventions are concrete and localised; and assessments are abstract and global, before intervening to centralise the M\&E function an assessment of the success or failure of the CCA programmes implemented thus far must be established. Equally important to note is that M\&E of CCA on the one hand and DRR on the other requires extensive, technical and scientific expertise which is lacking in South Africa. The UNFCC [20] argues that CCA and DRR are interdependent and can cause untold harm to human survival if one is treated differently from the other or as separate entities [20].

Nemakonde and Van Niekerk [32] explain that DRR and CCA have developed independently in most of the SADC countries and the common aim is to reduce disaster risks and poverty levels, save lives and protect infrastructure. The SADC countries also acknowledge that DRR and CCA involve all other sectors, individuals and communities in one way or another, yet they function independently of each other [31].

In any event, the International Strategy for Disaster Reduction recommends that by integrating and coordinating DRR policies and CCA programmes, weather-related disasters can be substantially reduced [12]. It is for this purpose that CCA and DRR must use the same resources and expertise to achieve the same objectives. Therefore, the centralisation of the M\&E function of both these fields of study will yield enough data to support the view that they must operate as a single entity [12], [15].

Similarly, the NDMF [27] discusses the benefits of cluster groups working together in the CCA planning process and states that by working together, consistency between statutory and strategic planning is enhanced; instead of a one-method-fits-all approach, a multi-risk level is encouraged; various government departments take responsibility for successes and challenges; and, conflict is minimised, since competing interests are prioritised by sharing resources and responsibilities [32].

\subsection{Recommendation 3: Formation of a CCA forum}

The second recommendation is that a CCA forum is established in South Africa. Although the HFA [24] provides guidelines for the effective functioning of this forum, many countries are still grappling with the formulation of their DRR plans. However, in South Africa, DRM plans, policies and frameworks are of international standards and this includes well- 
established disaster risk-related organisations within its borders. For this reason, it will be very easy to establish a climate change forum in SA [24].

However, the national and provincial DRM advisory forums are functioning at low levels. The reason for this low level of functioning may be attributed to lack of skills, funding and other resources. To add to this dilemma, the members of these advisory forums are officials from within their own ranks and do not want to be negatively critical of senior management in their departments. Instead of working as support structures and offering constructive criticisms for effective service delivery, reports for specific periods are presented in these advisory forum meetings. This reporting does not allow for effective growth and development of the disaster management centres and thus poor service delivery is the norm. Thus, this paper suggests the formation of an independent body (climate change forum) which has a vested interest in holding government to account for inefficiencies, relating to CCA. In as much as The Hyogo Framework for Action provides broad guidelines for the formation and functioning of this forum, it must be institutionally supported with adequate resources [24].

Finally, such a forum must be an all-inclusive, community-based initiative. It must include stakeholders from the four designated areas: government, private sector, non-governmental organisations and institutions of higher education and learning. The responsibility of higher education institutions in this forum is to assist with the development of effective policies based on scientific research. In fact, the forum must be participatory and community-based and receive the necessary political support to develop scientific and technical expertise In brief, the main purpose of this forum should be to agitate for the successful implementation of effective CCA policies and programmes to reduce poverty levels through sustainable development.

\subsection{Recommendation 4: Local community and development}

Global warming and climate change increase human fragility, which is worsened by the HIV/AIDS pandemic, gender inequalities, food insecurities and a general lack of quality education. This fragility affects most impoverished and vulnerable communities whose livelihood is always threatened. To strengthen the livelihood of the vulnerable communities, a community-based collaborative approach must be applied to ensure that developmental activities become sustainable. By applying a community-based collaborative approach, the resources from within communities may be harnessed. In this way, their exposure to hazards may be better understood which is very important for effective DRR [33].

Equally important is that most humanitarian organisations work with local communities at grass-roots level. They understand that communities possess a wealth of knowledge regarding issues such as CCA. For this reason, community-based organisations must be involved for successful implementation of CCA programmes. As a matter of fact, humanitarian organisations must also be involved to ensure buy-in and ownership, especially when developing CCA strategies, which is a relatively new phenomenon in South Africa.

\section{CONCLUSION}

Extensive CCA research has been carried out with many susceptible communities in many parts of the world with extensive evidence showing that global warming leads to climate change which impacts negatively on communities and infrastructure. In addition, global warming is anthropogenic and therefore with concerted effort it may be reversed if effective behaviour modification programmes are implemented and monitored. For this reason, behaviour change of communities must be considered a priority by all governments. 
The development of the South African NCCR White Paper in the year 2011 is a relatively new field in SA which is a new dimension to the M\&E of DRR within the global warming/climate change context. Whilst CCA seeks to address systemic changes to reduce the main causes of global warming, DRR addresses ongoing community challenges. The M\&E of DRR and CCA are skill and finance intensive and the implications of inefficiency for both fields of study are enormous. In addition, GHG emissions, global warming and climate change, all place undue strain on governments that are already overburdened. For these reasons, concerted efforts must be made by all countries to reduce the impact of climate change and especially hydrometeorological disasters.

The second section presents an overview of the conceptualisation of DRM and climate change. In the third section a discourse on the methodology for this research paper is presented. The fourth section presents an analysis and discussion of the results and the arguments for the praxis of climate change in the context of DRM. This is followed by the conclusion undertaken for this research which advocates for the deliberate implementation of behaviour modification programmes to promote CCA as an important climate change response strategy for South Africa. In addition, the effectiveness of this programme will be enhanced if it is supported by an effective monitoring, evaluation and reporting mechanism.

There is a sudden increase in DRR and climate change research which has led to a body of knowledge which is fragmented. This paper has provided for a new thinking of the convergence of both fields - climate change focuses on future related policy matters relating to preventative measures whilst DRR focuses on planning for and mitigating against the onslaught of the adverse impacts of hazards.

Thus, the recommendations presented in this paper must be presented to various constituencies for adoption and consequently implementation.

\section{REFERENCES}

[1] Van der Waldt, G. et al., Municipal Management: Serving the People, Juta and Company: Cape Town, 2007.

[2] Vermaak, J. \& Van Niekerk, D., DRR initiatives in South Africa. Journal Development Southern Africa, 21(3), pp. 555-574, 2004.

[3] Coburn, S. \& Promonis, Municipal Management: Serving the People, eds G. Van der Waldt et al., Juta and Company: Cape Town, 2007.

[4] RSA (Republic of South Africa), Disaster Management Act (Act No. 57 of 2002), Pretoria: Government Printer, 2003.

[5] Wisner, B., Blaikie, P., Cannon, T. \& Davis, I., At Risk: Natural Hazards, People’s Vulnerability and Disasters, 2nd ed., Routledge, 2007.

[6] Mercer, J., Disaster risk reduction or climate change adaptation: Are we reinventing the wheel? Journal of International Development: The Journal of the Development Studies Association, 22(2), pp. 247-264, 2010.

[7] United Nations Development Programme (UNDP), Designing CCA Initiatives: A UNDP Instrument kit for Practitioners, 2010.

[8] Phelan, C. \& Wren, J., Exploring reliability in academic assessment, 2007. https://chfasoa.uni.edu/reliabilityandvalidity.htm. Accessed on: May 2017.

[9] Creswell, J., Qualitative Inquiry and Research Design, 3rd ed., SAGE: Los Angeles, 2013.

[10] Gliem, J.A. \& Gliem, R.R., Calculating, interpreting, and reporting Cronbach's alpha reliability coefficient for Likert-type scales. Midwest Research-to-Practice Conference in Adult, Continuing, and Community Education, 2003. 
[11] SALGA (South African Local Government Association), Disaster Risk Management Status Assessment at Municipalities in South Africa, Pretoria, 2011.

[12] United Nations, International Strategy for Disaster Reduction (UNISDR), Global Assessment Report on DRR, United Nations: Geneva, 2009.

[13] UNISDR (United Nations International Strategy for Disaster Reduction), National Platform for DRR, Geneva, 2007.

[14] Spearman, M. \& McGray, H., Making Adaptation Count: Concepts and Resources for Monitoring and Evaluation of Climate Change Adaptation, World Resources Institute: Washington, DC, 2011.

[15] RSA (Republic of South Africa), National Climate Change Response, Government Printer: Pretoria, 2011.

[16] Wold, C., Hunter, D. \& Powers, M., Climate Change and the Law, 2nd ed., LexisNexis Matthew Bender, 2013.

[17] Climate and Development Knowledge Network, Managing climate extremes and disasters in Africa: Lessons from the SREX Report, CDKN, 2012.

[18] SADC (Southern African Development Community), Climate Change and Adaptation in SADC: A Strategy for the Water Sector, Gaborone, 2011.

[19] World Resources Institute (WRI), South Africa: An Experiment in CCA Planning, Washington, DC, 2009.

[20] United Nations Convention Framework on Climate Change (UNFCC), Handbook, Bonn, Germany, 2006.

[21] Izrael, Y.A., The Fourth Assessment Report of the Intergovernmental Panel on Climate Change, Working Group II, vol. 3, issue 9, pp. 551-556, 2007.

[22] Smith, J.B. \& Lenhart, S.S., Climate change adaptation policy options. Climate Research, 6(2), pp. 193-201, 1996.

[23] Department for International Development, www.gov.uk/gov. Accessed on: 10 Jul. 2017.

[24] UNISDR (United Nations International Strategy for Disaster Reduction), Hyogo Framework for Action (HFA): 2005-2015, Geneva, 2005.

[25] UNISDR (United Nations International Strategy for Disaster Reduction), African Regional Strategy for DRR and Plan of Action, Geneva, 2004.

[26] UNOCHA (United Nations Office for the Co-ordination of Human Affairs), Draft South African Development Community (SADC) Regional DRR Strategy, Geneva, 2011.

[27] RSA (Republic of South Africa), National Disaster Management Framework for Disaster Risk Management in South Africa, Government Printer: Pretoria, 2005.

[28] PMG (Parliamentary Monitoring Group), Disaster Management Centre Operations and How its Funds Are Distributed, Cape Town, South Africa, 2012.

[29] Riekert, S.P., The requirements for the development of a spatial information system for the Tlokwe Local Municipality water catchments area. Master's thesis, North-West University, Potchefstroom, 2014.

[30] Davies, J., Policies in support of pastoralism and biodiversity in the heterogeneous drylands of East Africa. Pastoralism: Research, Policy and Practice, 2(14), 2012.

[31] IFRC and Red Crescent Societies (IFRC), DREF Final Report South Africa: Floods. Department of Cooperative Governance, National Disaster Management Centre, Annual Report 2014-2015.

[32] Nemakonde, L.D. \& Van Niekerk, D., A normative model for integrating organisations for DRR and CCA within SADC member states. Disaster Prevention and Management: An International Journal, 26(3), pp. 361-337, 2017. 